\title{
Correction: Multi-trait analysis for genome-wide association study of five psychiatric disorders
}

Yulu Wu, Hongbao Cao, Ancha Baranova, Hailiang Huang, Sheng Li, Lei Cai, Shuquan Rao, Minhan Dai, Min Xie,

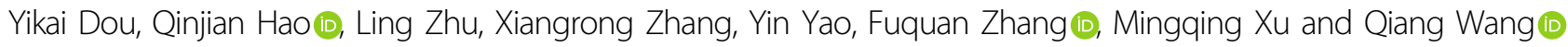

Correction to: Translational Psychiatry https://doi.org/10.1038/s41398-020-00902-6 published online 11 July 2020

The original version of this Article was updated shortly after publication to correct the order of the corresponding authors. The correct order should be: Fuquan Zhang, Mingqing $\mathrm{Xu}$ and Qiang Wang.

Published online: 14 July 2020 